\title{
On determining if a specular point exists
}

\author{
Rusch, W; Sørensen, 0
}

Published in:

I E E E Transactions on Antennas and Propagation

Link to article, DOI:

10.1109/TAP.1979.1142021

Publication date:

1979

Document Version

Publisher's PDF, also known as Version of record

Link back to DTU Orbit

\section{Citation (APA):}

Rusch, W., \& Sørensen, O. (1979). On determining if a specular point exists. I E E E Transactions on Antennas and Propagation, 27(1), 99-101. https://doi.org/10.1109/TAP.1979.1142021

\section{General rights}

Copyright and moral rights for the publications made accessible in the public portal are retained by the authors and/or other copyright owners and it is a condition of accessing publications that users recognise and abide by the legal requirements associated with these rights.

- Users may download and print one copy of any publication from the public portal for the purpose of private study or research.

- You may not further distribute the material or use it for any profit-making activity or commercial gain

- You may freely distribute the URL identifying the publication in the public portal

If you believe that this document breaches copyright please contact us providing details, and we will remove access to the work immediately and investigate your claim. 


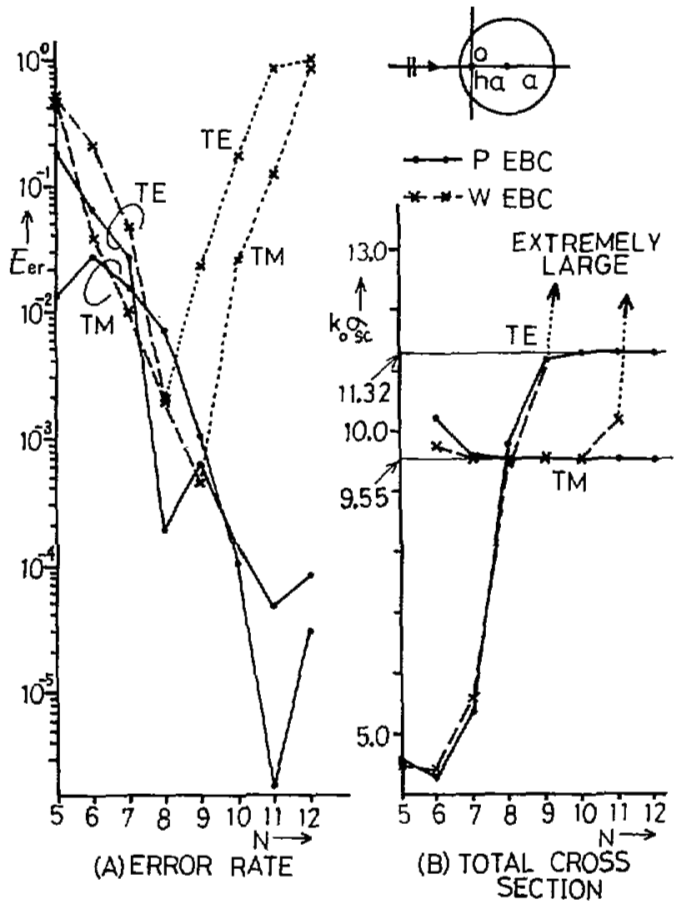

Fig. 3. Comparison between the proposed EBC and Waterman's EBC for an origin-shifted circular cylinder $\left(k_{0} a=2.0, h=0.6\right.$, $\kappa=3.0$ ).

trophe in an earlier stage for the cases where the degree of convergence of the series solution is not so good. It is to be mentioned that the proposed method does not always give results superior to Waterman's and on the contrary in some cases of near-circular cross section shape Waterman's method happens to give better results. As for the computing time, the proposed method consumes more time than Waterman's method, but not so much as expected from a simple consideration of the number of multiplications and divisions. For example, it is only increased 30 percent at most in the case of $N=16$ of Fig. 2. (The total computing time for this example is about $23 \mathrm{~s}$ on the NEAC 2200-700 computer.)

The EBC method is simpler and more useful than the method of solving directly the integral equations [6, eqs. (43)(46) or (53)-(56)] when the boundary shape of the dielectric cylinder is smooth and convex. For the dielectric cylinder of relatively complex boundary shape, however, the limitation of applicability of the EBC method seems to exist.

\section{REFERENCES}

[1] P. C. Waterman, "Scattering by dielectric obstacles," Alta Freq., vol. 38 (Speciale), pp. 348-352, 1969.

[2] P. W. Barber and C. Yeh, "Scattering of electromagnetic waves by arbitrarily shaped dielectric bodies," Appl. Opt., vol. 14, pp. 2864-2872, Dec. 1975.

[3] P. W. Barber, "Resonance electromagnetic absorption by nonspherical dielectric objects," IEEE Trans. Microwave Theory 1ech., vol. MTT-25, pp. 373-381, May 1977.

[4] T. K. Wu and L. L. Tsai, "Numerical analysis of electromagnetic fields in biological tissues," Proc. IEEE (Letters), vol. 62, pp. 1167-1168, Aug. 1974.

[5] -, "Electromagnetic fields induced inside arbitrary cylinders of biological tissue," IEEE Trans. Microwave Theory Tech., vol. MTT-25 (Short Papers), pp. 61-65, Jan. 1977.

[6] N. Morita, "Surface integral representations for electromagnetic scattering from dielectric cylinders," IEEE Trans. Antennas Propagat., vol. AP-26, pp. 261-266, Mar. 1978.

[7] K. Yasuura, Inst. Elec. Eng. Japan, Tech. Rep. EMT-76-31, Oct. 1976.

[8] J. Van Bladel, Electromagnetic Fields. New York: McGrawHill, 1964, p. 373.

\section{On Determining if a Specular Point Exists}

\author{
W. V. T. RUSCH, FELLOW, IEEE AND O. SØRENSON
}

\begin{abstract}
A technique is presented wherel,y the existence of a specular point on a convex surface of revolution can be determined without actually finding it. Only the evaluation of two simple algebraic expressions is involved. Should a specular point be found not to exist, a search procedure has been thereby eliminated.
\end{abstract}

\section{INTRODUCTION}

All geometrical theories of diffraction require superposition of a classical specular or geometrical-optics ray to determine the total field. Geometrical laws relate the reflected and incident fields at a specular point in a straightforward manner [1], yet the location of a specular point on a complex surface can be a difficult, or at least tedious, procedure. Depending on the reflector geometry, and the location of the source and field points, one- or two-dimensional iteration schemes can be used. These techniques waste time and yield ambiguous results, however, if the source/field point orientation is such that a specular point does not exist.

A procedure based on Fermat's principle will be described whereby the existence of a specular point can be determined for a general convex surface of revolution. The following two cases are considered.

1) Source and field point are coplanar with the symmetry axis.

2) Source and field point location is unrestricted.

\section{ANALYTIC TREATMENT}

Let $O$ be the source point, $P$ be the observation point, $R$ be a point on the surface, $\overline{O R}$ be the vector from $O$ to $R$, and $\overline{R P}$ be the vector from $R$ to $P$ (Fig. 1). Let $R_{0}$ be a specular point on the surface. Define

$$
d \equiv|\widehat{O R}|+|\widehat{R P}| .
$$

For a convex monotonic surface $d$ will be a global minimum at $R_{0}$ so that, at most, one specular point may exist on the surface. The gradient of $d$ with respect to the coordinates of $R$ is

$$
\nabla_{R} d=\frac{\overline{O R}}{|\overline{O R}|}-\frac{\overline{R P}}{|\overline{R P}|}
$$
1978.

Manuscript received September 16, 1977; revised February 15, W. V. T. Rusch is with the Department of Electrical Engineering, University of Southern California, Los Angeles, CA 90007.

O. Sørenson was with the Electromagnetics Institute, Technical University of Denmark. 


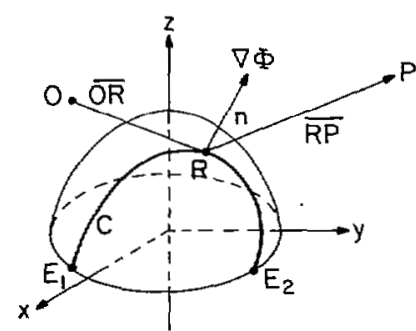

Fig. 1. Geometry of general problem.

and it is easily shown that

$$
\nabla_{R} d \cdot(\overline{O R} \times \overline{R P})=0 .
$$

Thus $\nabla_{R} d$ lies in the plane containing $O, R$, and $P$. Next, define

$$
\bar{v} \equiv \nabla_{R} d-\left(\nabla_{R} d \cdot \hat{n}\right) \hat{n}
$$

where $\hat{n}$ is the outward normal at $R$. Thus $\bar{v}$ is the projection of $\nabla_{R} d$ on a plane tangent to the surface at $R$, and $\bar{v}$ also lies in the plane containing $O, R$, and $P$. For $R$ in the vicinity of $R_{0}, \vec{v}$ will be directed away from $R_{0}$ since $d$ is a minimum at $R_{0}$.

\section{A. Axially Symmetric Convex Reflector with Source and Observation Point in the Same Aximuthal Plane}

The planar cross section containing $O, P$, and the reflector axis is shown in Fig. 2. The specular point $R_{0}$ will also lie in this plane. Consequently, this special case reduces to a two-dimensional problem.

As shown in the previous section, for all points $R$ in the planar cross section, $\bar{v}$ also lies in this plane and can only be zero at a specular point which may or may not exist. Furthermore, if a specular point exists, as shown in the figure, $\bar{v}$ will point to the right for all $R$ on the right side of $R_{0}$ and to the left for all $R$ on the left of $R_{0}$. Thus, at both edges of the surface in the cross section ( $E_{1}$ and $E_{2}$ ), $\bar{v}$ will point away from the surface. However, if no specular point exists, $v$ cannot reverse direction (because it will be necessary to become zero in the process). Consequently, $\bar{v}$ will point in the same direction along the entire surface and will point toward the surface at one edge and away from it at the other.

Thus, for this two-dimensional special case, in order to determine if a specular point exists, $\bar{v}$ need only be evaluated at the two edges. The specular point will exist if and only if $\vec{v}$ points away from the surface at both edges. If a specular point is found to exist, many different algorithms are available to locate it, one of which may be based on the principles of this communication [2].

\section{B. Axially Symmetric Convex Reflector with Source and Observation Point Not Restricted to the Same Azimuthal Plane}

The geometry of this configuration is shown in Fig. 1. Define the surface by

$$
\begin{aligned}
\rho^{2} & =f(z) \\
\Phi(\rho, z) & =\rho^{2}-f(z)=0 \\
\nabla \Phi & =2 x \hat{a}_{x}+2 y \hat{a}_{y}-f^{\prime}(z) \hat{a}_{z} .
\end{aligned}
$$

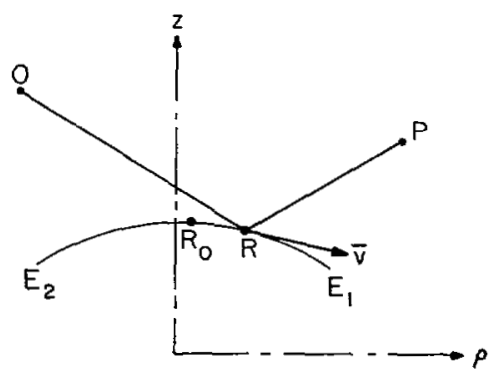

Fig. 2. Geometry of planar problem.

In this case $O$ and $P$ do not necessarily lie in the same azimuthal plane. However, according to Fermat's Principle, at the specular point, $O, R, P$, and $\nabla_{R} \Phi$ (or $\hat{n}$ ) all lie in the same plane. Hence

$$
\nabla_{R} \Phi \cdot(\overline{O R} \times \overline{R P})=0 .
$$

Expanding (8) yields

$$
A x_{R}+B y_{R}+C=0
$$

where

$$
\begin{aligned}
A= & -2\left[\left(y_{O}-y_{P}\right)\left(z_{R}-z_{O}\right)+y_{O}\left(z_{O}-z_{P}\right)\right] \\
& -f^{\prime}\left(z_{R}\right)\left(y_{O}-y_{P}\right) \\
B= & +2\left[\left(x_{O}-x_{P}\right)\left(z_{R}-z_{O}\right)+x_{O}\left(z_{O}-z_{P}\right)\right] \\
& +f^{\prime}\left(z_{R}\right)\left(x_{O}-x_{P}\right) \\
C= & f^{\prime}\left(z_{R}\right)\left(x_{P} y_{O}-y_{P} x_{O}\right) .
\end{aligned}
$$

Equations (9)-(12) represent a three-dimensional surface $S$, which reduces to the cross-sectional plane of the previous section when $x_{O} / y_{O}=x_{P} / y_{P}$.

The surface $S$ intersects the axially symmetric reflector in a curve $C$ as shown in the figure. The specular point $R_{0}$, if it exists, lies along $C$. At every point $R$ along $C, \vec{v}$ is tangent to $C$. Consequently, as in the previous section, the problem reduces to finding the two points $E_{1}$ and $E_{2}$ where $C$ intersects the reflector rim and to evaluating $\bar{v}$ at those points. A specular point will exist if and only if $\bar{v}$ points away from the surface at both $E_{1}$ and $E_{2}$.

The two edge points may be found by substituting

$$
x_{R}{ }^{2}+y_{R}^{2}=f\left(z_{\mathrm{RIM}}\right)
$$

into (9). The four possible types of solution are as follows.

1) If $B=O$ :

$$
\begin{aligned}
& x_{\mathrm{RIM}}=-C / A \\
& y_{\mathrm{RIM}}= \pm \sqrt{f\left(z_{\mathrm{RIM}}\right)-(C / A)^{2}} . \\
& \text { 2) If } A=O: \\
& y_{\mathrm{RIM}}=-C / B \\
& x_{\mathrm{RIM}}= \pm \sqrt{f\left(z_{\mathrm{RIM}}\right)-(C / A)^{2}} .
\end{aligned}
$$

3) If $A=O$ and $B=O: O$ and $P$ are coincident, causing (9) to become indeterminant. However, the techniques of the previous section can be used. 
4) If $A \neq O, B \neq O$ :

$$
\begin{gathered}
y_{\mathrm{RIM}}=-\frac{A}{B} x_{\mathrm{RIM}}-\frac{C}{B} \\
x_{\mathrm{RIM}}{ }^{2}\left[1+\left(\frac{A}{B}\right)^{2}\right]+\left[\frac{2 A C}{B^{2}}\right] x_{\mathrm{RIM}} \\
+\left[\frac{C^{2}}{B^{2}}-f\left(z_{\mathrm{RIM}}\right)\right]=0 .
\end{gathered}
$$

The descriminant of $(16 \mathrm{~b})$ is never negative so that two real roots always exist.

As a specific example, let $x_{O}=x_{P}=O$ so that both $O$ and $P$ lie in the $y z$ plane. Then, from (11) and (12), $B=C=$ $O$ so that (14a) and (14b) yield $x_{R I M}=O$ and $y_{R I M}=$ $\pm \rho_{R I M}$, as expected.

Having, again, as in the previous section, demonstrated the existence of a specular point for the nonplanar case, many different search techniques are available for locating it. However, should the simple evaluation of $\bar{v}$ at the two points on the reflector ring defined by (9) indicate that no specular point exists, a search procedure has been eliminated.

\section{REFERENCES}

[1] S. W. Lee, "Electromagnetic reflection from a conducting surface: Geometrical optics solution," IEEE Trans. Antennas Propagat., vol. AP-23, Mar. 1975.

[2] W. V. T. Rusch and O. Sфrenson, "On determining if a specular point exists," in Proc. URSI Symp. Electromagnetic Wave Theory, 1977, pp. 284-286.

\section{Transient and Time-Harmonic Dyadic Green's Functions for a Perfectly Conducting Cone}

\section{K. K. CHAN AND L. B. FELSEN}

Abstract-New representations for the time-dependent dyadic Green's functions for a perfectly conducting semi-infinite cone are presented. For the special case of small cone angles and an on-axis source, simplified expressions are given for both the time-dependent and time-harmonic regimes.

In a separate paper, new representations for the time-dependent scalar Dirichlet and Neumann Green's functions for a semi-infinite cone were developed [1]. It was shown that these formulations can be simplified substantially when the cone angle is small and the source is located on the cone axis. It was also shown that new closed-form time-harmonic solutions can be obtained by Fourier transformation of the transient fields.

Manuscript received October 17, 1977.

K. K. Chan is with the National Chiao-Tung University, Hsinchu, Taiwan.

L. B. Felsen is with the Polytechnic Institute of New York, Farmingdale, NY 11735.
The present communication extends these results to the dyadic Green's functions generated by vector dipole excitation.

Consider an electric dipole of vector strength $\mathbf{J}$ situated at the point $\mathbf{r}=\mathbf{r}^{\prime}$ outside a perfectly conducting cone defined by the surface $\theta=\theta_{0}$. The components of $\mathbf{J}$ in the $(r, \theta, \phi)$ directions are $J_{r}, J_{\theta}$, and $J_{\phi}$, respectively. The total field can be regarded as a superposition of the separate responses due to $J_{r}, J_{\theta}$, and $J_{\phi}$. The response to a radial electric dipole source may be obtained from the scalar Dirichlet Green's function discussed in [1]. The electric field due to a transverse dipole source of vector strength $\mathbf{J}_{t}$ can be represented in terms of functions $S^{\prime}$ and $S^{\prime \prime}$ as follows [2] :

$$
\begin{aligned}
\mathbf{E}= & E_{\mathrm{r}_{\mathrm{r}}}+E_{\theta} \theta_{0}+E_{\phi} \phi_{0} \\
E_{r}= & \left(\frac{\partial^{2}}{\partial r^{2}}+k^{2}\right)\left(\mathbf{J}_{t} \cdot{ }_{t} \nabla^{\prime}\right) \frac{\partial}{\partial r^{\prime}}\left(\frac{S^{\prime}}{-i \omega \epsilon}\right) \\
E_{\theta}= & \frac{-1}{i \omega \epsilon r} \frac{\partial^{2}}{\partial r \partial \theta}\left(\mathbf{J}_{t} \cdot{ }_{t} \nabla^{\prime}\right) \frac{\partial s^{\prime}}{\partial r^{\prime}} \\
& -\frac{i \omega \mu}{r \sin \theta} \frac{\partial}{\partial \phi}\left(\mathbf{J}_{t} \times \mathbf{r}_{0} \cdot{ }_{t} \nabla^{\prime} S^{\prime \prime}\right) \\
E_{\phi}= & -\frac{1}{i \omega \epsilon r \sin \theta} \frac{\partial^{2}}{\partial r \partial \phi}\left(\mathbf{J}_{t} \cdot{ }_{t} \nabla^{\prime}\right) \frac{\partial S^{\prime}}{\partial r^{\prime}} \\
& +\frac{i \omega \mu}{r} \frac{\partial}{\partial \theta}\left(\mathbf{J}_{t} \times \mathbf{r}_{0} \cdot{ }_{t} \nabla^{\prime} S^{\prime \prime}\right)
\end{aligned}
$$

where

$$
\begin{gathered}
\mathbf{J}_{t} \cdot{ }_{t} \nabla^{\prime}=\frac{J_{\theta}}{r^{\prime}} \frac{\partial}{\partial \theta^{\prime}}+\frac{J_{\phi}}{r^{\prime} \sin \theta^{\prime}} \frac{\partial}{\partial \phi^{\prime}} \\
\mathbf{J}_{t} \times \mathbf{r}_{0} \cdot{ }_{t} \nabla^{\prime}=\frac{J_{\phi}}{r^{\prime}} \frac{\partial}{\partial \theta^{\prime}}-\frac{J_{\theta}}{r^{\prime} \sin \theta^{\prime}} \frac{\partial}{\partial \phi^{\prime}} .
\end{gathered}
$$

The scalar functions $S^{\prime}, S^{\prime \prime}$ can be decomposed into a freespace portion and a perturbation term accounting for the effect of the cone. Since the free-space portion of the solution is known, only the evaluation of the perturbation fields (distinguished by subscript $s$ ) need concern us here. Suitable representations for the latter are [3]

$$
\begin{aligned}
S_{s}{ }^{\prime}= & S_{s_{1}}{ }^{\prime}+S_{s_{2}}{ }^{\prime} \\
S_{s_{1}}{ }^{\prime}= & -\frac{i}{2 \pi k} j_{0}\left(k r_{1}\right) h_{0}{ }^{(1)}\left(k r_{2}\right) \sum_{m=1}^{\infty} \frac{\cos m\left(\phi-\phi^{\prime}\right)}{m} \\
& \cdot\left[\tan \frac{\theta}{2} \tan \frac{\theta^{\prime}}{2} \tan ^{2}\left(\frac{\pi-\theta_{0}}{2}\right)\right]^{m} \\
S_{s_{2}}{ }^{\prime}= & \frac{1}{8 \pi k} \sum_{m=0}^{\infty} \epsilon_{m} \cos m\left(\phi-\phi^{\prime}\right) \int_{-1 / 2-i \infty}^{-1 / 2+i \infty} d v(2 v+1) \\
& \cdot \frac{h_{v}^{(1)}(k r) h_{v}(1)\left(k r^{\prime}\right)}{v(v+1)} \frac{P_{v}-m(\cos \theta) P_{v}-m\left(\cos \theta^{\prime}\right)}{\sin (v-m) \pi} \\
& \cdot \frac{\Gamma(v+m+1)}{\Gamma(v-m+1)} \frac{P_{v}{ }^{-m}\left(-\cos \theta_{0}\right)}{P_{v}{ }^{-m}\left(\cos \theta_{0}\right)}
\end{aligned}
$$

